\title{
A simple mechanistic model of sprout spacing in tumour-associated angiogenesis
}

\author{
B. Addison-Smith ${ }^{\mathrm{a}, *}$, D.L.S. McElwain ${ }^{\mathrm{a}}$, P.K. Maini ${ }^{\mathrm{b}, \mathrm{c}}$ \\ ${ }^{\text {a }}$ School of Mathematical Sciences, Queensland University of Technology, GPO Box 2434, Brisbane 4001, Australia

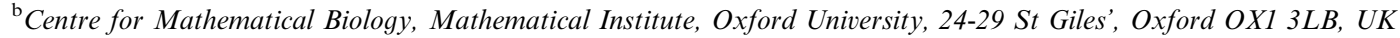 \\ ${ }^{\mathrm{c}}$ Oxford Centre for Integrative Systems Biology, Department of Biochemistry, South Parks Road OX1 3QU, UK
}

Received 19 September 2006; received in revised form 4 July 2007; accepted 14 August 2007

Available online 7 September 2007

\begin{abstract}
This paper develops a simple mathematical model of the siting of capillary sprouts on an existing blood vessel during the initiation of tumour-induced angiogenesis. The model represents an inceptive attempt to address the question of how unchecked sprouting of the parent vessel is avoided at the initiation of angiogenesis, based on the idea that feedback regulation processes play the dominant role. No chemical interaction between the proangiogenic and antiangiogenic factors is assumed. The model is based on corneal pocket experiments, and provides a mathematical analysis of the initial spacing of angiogenic sprouts.
\end{abstract}

(C) 2007 Elsevier Ltd. All rights reserved.

Keywords: Angiogenesis; Mathematical model; Inhibitor; VEGF; Endostatin

\section{Introduction}

In any cellular environment, precise execution of activities depends on well-coordinated control mechanisms. Feedback inhibition is one of the methods by which such control may be achieved; an enzyme which catalyses a primary reaction may have this action inhibited by one of the downstream products of the reaction. We propose that such a mechanism is involved in the regulation of vascular sprout formation during tumour-induced angiogenesis, and develop a simple mechanistic mathematical model to describe the determination of sprouting sites along an existing blood vessel.

Angiogenesis, the growth of new blood vessels from an existing vasculature, is a critical process in the formation of solid tumours. Before reaching an angiogenic stage, such tumours may exist for some time as dormant, avascular, masses of limited size (up to approximately $2 \mathrm{~mm}$ in diameter). Growth of these masses is restricted by the need to obtain oxygen and nutrients by diffusion. If the tumorous mass switches to an angiogenic phenotype, a

\footnotetext{
${ }^{*}$ Corresponding author. Tel.: + 61738644442 ; fax: +61738642310.

E-mail address: b.addison-smith@qut.edu.au (B. Addison-Smith).
}

new blood vessel network is formed between the existing vasculature and the tumour, providing a source for oxygen and nutrients. Rapid growth of the tumour is consequently enabled. Formation of the new blood vessel network is guided by a complex interplay of both pro- and antiangiogenic molecules produced by a variety of sources including tumour cells, endothelium, extracellular matrix, pericytes, and plasma clotting products (see, for review, Araujo and McElwain, 2004; Mantzaris et al., 2004). This paper is concerned with the products and processes involved in the initiation of angiogenic sprouting. We present a mathematical model based on the hypothesis that sprouting is initiated by a threshold concentration of angiogenic growth factor, and that antiangiogenic products synthesized in the immediate vicinity of each new sprout will act locally to prevent the overwhelming formation of new sprouts. This feedback inhibition process is similar to that described by Karihaloo et al. (2001) in which it is proposed that the antiangiogenic factor, endostatin, plays a role in preventing unchecked outgrowth of the growing ureteric bud.

Previous modelling work includes that of Orme and Chaplain (1996), who developed a model of sprout formation which is based upon phenomena occurring 
within the parent vessel. They develop a three species model: endothelial cells, matrix, and adhesive sites. Using this model and a stability analysis, they argue that a natural spacing appears with the space between sprouts determined from these equations in a manner similar to Turing instabilities. They do not include any feedback mechanism. Levine et al. (2001) have also developed a model for the onset of capillary formation. Their model, based upon the theory of reinforced random walks, uses the assumption that angiogenic factor is transformed into a proteolytic enzyme that enables a sprout to form. Their model makes predictions about the aggregation of endothelial cells, and the perforation of the basement membrane that allows the formation of a new sprout. Again there is no feedback of the form discussed in our current work.

An alternative approach to vascular network growth modelling is adopted by Gazit (1996, Chapter 5), who uses numeric simulations on a square lattice, typically $128 \times 128$. The network is initiated with a single seed and then extends in response to a diffusible growth factor produced by a source outside the network. Optionally, local amplification can occur at an activated network site and this, in turn, produces further diffusible growth factor.

Before presenting the mathematical model we discuss antiangiogenic products which are found in the vicinity of the tumour-induced angiogenesis, and which may act to influence the patterns of sprouting.

\subsection{Formation of antiangiogenic molecules during tumour- induced angiogenesis}

The search for endogenous antiangiogenic molecules produced within the microenvironment of a tumour was initially prompted by observations that excision of a primary tumour can often lead to rapid growth of its previously dormant metastases. The suppression of secondary growths was thought to be due to antiangiogenic factors, produced in the environment of the main tumour, but acting at the site of distant metastases to prevent these small masses of tumour attaining vascularisation. Although proangiogenic factors are also produced by the primary tumour, it was suggested that the antiangiogenic molecules had a longer half-life in circulation, and so became the dominant effect at the site of distant metastases. Researchers have subsequently uncovered many endogenous inhibitors of angiogenesis, including tumstatin, arrestin, and canstatin, and most notably, angiostatin and endostatin. We will discuss angiostatin and endostatin briefly here. For recent reviews of these and other antiangiogenic molecules, see Clamp and Jayson (2005), Folkman (2005), Ruegg et al. (2006), Sim (1998).

Endostatin is an $18-22 \mathrm{kDa}$ fragment of collagen XVIII, which is notably present, amongst other collagens, in the vascular and epithelial basement membrane (Saarela et al., 1998). It has been shown to inhibit tumour-induced angiogenesis, and to inhibit endothelial cell (EC) prolifera- tion and migration, with the possible mechanism being disruption of cell-matrix interactions. Cleavage of endostatin from collagen XVIII is mediated by some of the matrix metalloproteases (MMPs) (Heljasvaara et al., 2005), and, at low $\mathrm{pH}$, by the protease, cathepsin $\mathrm{L}$, independent of MMP activity (Felbor et al., 2000). Production of proteases such as cathepsin L and MMPs involved in the production of endostatin are known to be upregulated in the vicinity of tumour-induced angiogenesis (see, for instance, Taraboletti et al., 2002; Felbor et al., 2000; Jones et al., 1999). Additionally, it has been hypothesized that endostatin plays a role in preventing unchecked outgrowth of the developing ureteric bud. Karihaloo et al. (2001) proposed that MMPs degraded the basement membrane at the tip of the growing ureteric bud, resulting in an accumulation of endostatin which then acted to inhibit further branching. Felbor et al. (2000) also suggest that endostatin acts in a feedback mechanism during angiogenesis. We hypothesize that such a mechanism contributes to the prevention of unchecked sprouting from existing blood vessels during tumour-induced angiogenesis.

Other possible candidates for a feedback control mechanism of angiogenic sprouting include the molecule angiostatin. Angiostatin is a $38 \mathrm{kDa}$ protein, cleaved from the serum protein plasminogen by the action of such factors as tissue plasminogen activator (tPA) and several of the matrix metalloproteases (MMPs) (Jurasz et al., 2003). It has been shown to inhibit endothelial cell (EC) growth. During tumour-induced angiogenesis, growth factors secreted by the tumour initially cause hyperpermeability in nearby blood vessels (Dvorak, 2003). This occurs before spouting is initiated, before breakdown of the basement membrane, and results in leakage of the blood plasma into the surrounding extracellular matrix (ECM). This plasma contains both plasminogen, from which angiostatin is derived, and the factors which cleave plasminogen to form angiostatin, such as tissue plasminogen activator and MMPs. Tissue plasminogen activator and the MMPs may also be present in the ECM during angiogenesis through production by endothelial cells, or may be released by tumour cells. Thus angiostatin may be formed in the vicinity of blood vessels undergoing angiogenesis.

Sections 2 and 3 of this paper develop the mathematical model, Sections 4 and 5 present an analysis of the model with and without activator decay, results are discussed in Section 6, and Section 7 discusses conclusions and directions for future research.

\section{Model domain}

The domain of the mathematical model, shown in Fig. 1, is based on the physical set up of an experimental angiogenesis model known as the corneal pocket assay (see, for example, Gimbrone et al., 1974; Asahara et al., 1998). In these experiments a tumour fragment, or a pellet impregnated with a growth factor, such as vascular endothelial growth factor (VEGF), is implanted into 
a

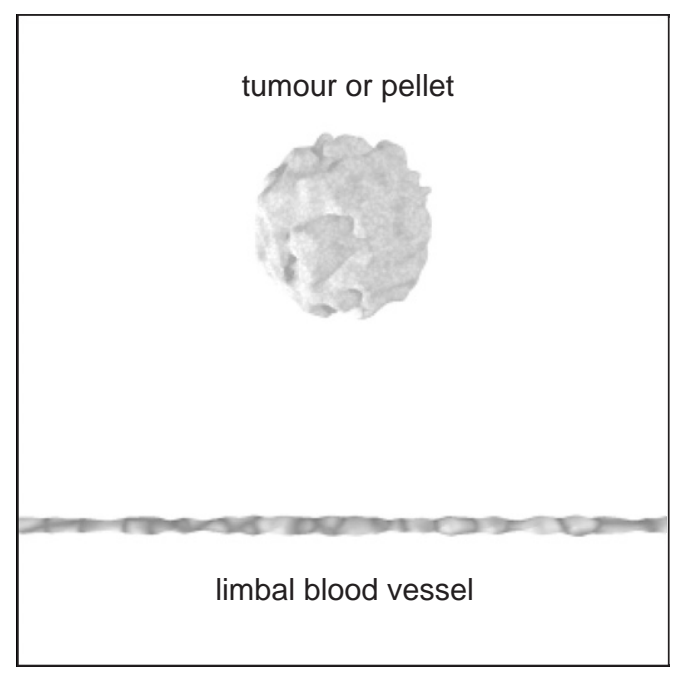

b

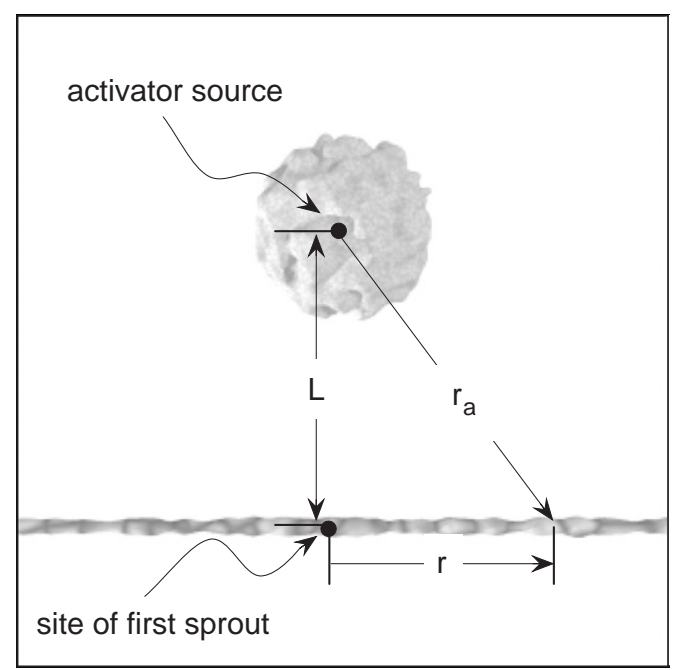

Fig. 1. Model domain: (a) schematic of elements of the corneal pocket assay; (b) measurement reference system.

a pocket created in the avascular cornea of a mouse or rabbit. The implant engenders an angiogenic response in nearby blood vessels - those in the limbus (periphery of the cornea) closest to the pocket. The progression of the angiogenic response through the normally avascular and semi-transparent cornea is relatively easy to inspect. Fig. 2 reproduces images from Asahara et al. (1998) showing angiogenic response in a mouse cornea 6 days after implantation with a pellet containing VEGF.

Observations of angiogenesis relevant to this paper from such assays can be summarized as follows: within a few hours of implantation, the nearby limbal blood vessels become hyperpermeable, and plasma leaks from the vessels into the surrounding stroma; endothelial cells that line the vessels produce enzymes which degrade the surrounding basement membrane; the cells begin to migrate through the gaps in the basement membrane, moving towards the
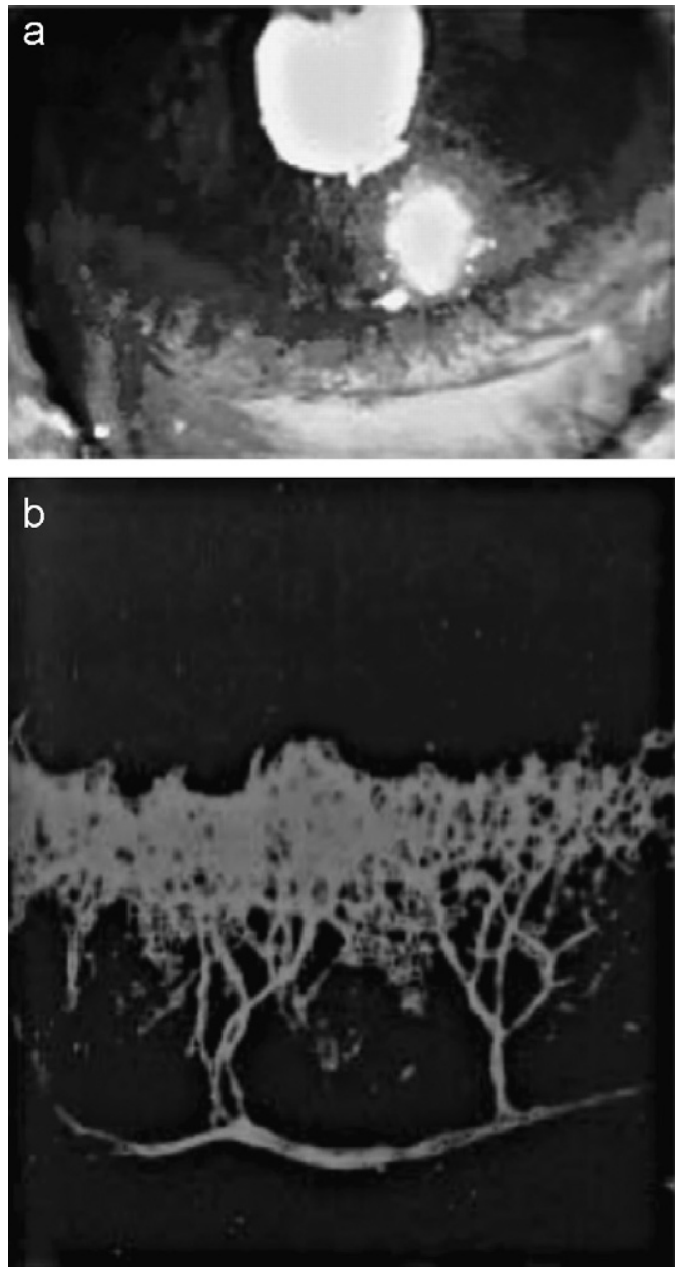

Fig. 2. Images of angiogenesis during a corneal pocket assay reproduced from Asahara et al. (1998), with permission: (a) Macroscopic photograph of angiogenesis in a mouse cornea 6 days after implantation of a VEGFimpregnated pellet during a corneal pocket assay. Reproduced from Asahara et al. (1998), with permission; (b) BS-1 lectin fluorescent staining of corneal limbal vessels undergoing angiogenesis in a corneal pocket assay. The image was taken 6 days after implantation of a pellet containing VEGF. Spacing of sprouts along the parent vessel is clear. The image also displays the brush-border effect associated with repeated capillary branching. Reproduced from Asahara et al. (1998), with permission.

source of angiogenic factor. Endothelial cell division is also upregulated.

Events such as endothelial cell migration and division are outside the scope of the investigation of the present paper as are further events such as tubular morphogenesis (ECs roll up and join together to form tubes) and anastomosis (tubes connect to form loops capable of circulating blood).

\section{Model development}

The tumour implant or pellet used in the corneal pocket assay is represented in our mathematical model by a point source, located a distance from the limbus corresponding to the centre of the implant (see Fig. 1). The limbus is 
modelled as a straight line. Fig. 1 shows the coordinate reference system used, noting that the mathematical model is developed here in cylindrical coordinates. The model incorporates two species: an angiogenesis activator (concentration denoted by A), such as VEGF, which is produced continuously by the tumour, and an angiogenesis inhibitor (concentration denoted by I), such as endostatin or angiostatin, which it is assumed is produced instantaneously at the initiation point of each new sprout. The species do not interact. This modelling allows for firstorder removal of the angiogenic activator from the system, representing either a natural decay process or other removal which can be represented by a first-order decay. For the purposes of this preliminary model, we assume that consumption or decay of the inhibitor is negligible, an assumption supported by observations, discussed is Section 1.1, of the apparent long-range effects of antiangiogenic factors on distant metastases. Sprouts may form at any point on the existing limbal blood vessel subject to the following criteria:

- the activator concentration at that point must be greater than or equal to a chosen trigger value, i.e. $A \geqslant A_{\text {trig }}$;

- the inhibitor concentration at that point must be less than or equal to a chosen inhibitor threshold, i.e. $I \leqslant I_{\text {thresh }}$.

The modelling begins as activator is released from the tumour into an empty domain. The first sprout occurs on the blood vessel at a point closest to the activator source, henceforth called the midpoint, and a 'puff' (instantaneous point source) of inhibitor is initiated. Subsequent sprouts occur symmetrically about the line connecting tumour and blood vessel, each new sprout adding an instantaneous point source of inhibitor.

\subsection{Governing equations for inhibitor and activator}

For the angiogenic factor, $A$, the governing equation is assumed to be

$\frac{\partial A}{\partial t}=D_{a} \nabla^{2} A-\lambda_{a} A$,

where $D_{a}$ is the diffusion coefficient, and $\lambda_{a}$ the decay coefficient, both taken as constant. Modelling the tumour as a continuous point source, we seek a solution of this equation in two dimensions in cylindrical coordinates, with radial symmetry. That is, we wish to solve Eq. (1) in an infinite 2D domain subject to the initial condition $A\left(r_{a}, 0\right)=0.0$, where $r_{a}$ is radial distance from the source, of strength $q$. This is found by integrating a decaying instantaneous point source solution with respect to time, to give,

$A\left(r_{a}, t\right)=\frac{q}{4 \pi D_{a}} \int_{0}^{t} \frac{1}{u} \exp \left(\frac{-r_{a}^{2}}{4 D_{a} u}-\lambda_{a} u\right) \mathrm{d} u$.
In the situations where decay is negligible, that is $\lambda_{a}=0$, the solution may be written as

$A\left(r_{a}, t\right)=\frac{q}{4 \pi D_{a}} E_{1}\left(\frac{r_{a}^{2}}{4 D_{a} t}\right)$

where $E_{1}(x)$ is a form of the special integral function $E_{n}(x)$ with $n=1$, given by

$E_{1}(x)=\int_{x}^{\infty}\left(\frac{\mathrm{e}^{-u}}{u}\right) \mathrm{d} u$

When decay is significant $\left(\lambda_{a} \neq 0\right)$, numeric integration must be used to evaluate expression (2).

Considering the system with activator decay, an expression for the activator steady state may be found in the form of a modified Bessel function of the second kind, namely,

$A\left(r_{a}\right)=\frac{q}{2 \pi D_{a}} K_{0}\left(r_{a} \sqrt{\frac{\lambda_{a}}{D_{a}}}\right)$.

There is no steady state for the activator without decay.

The concentration of inhibitor, $I$, subject only to Fickian diffusion, is governed by the conservation equation

$\frac{\partial I}{\partial t}=D_{i} \nabla^{2} I$,

where $D_{i}$ is the diffusion coefficient, assumed constant.

To model an instantaneous point source of inhibitor in two-dimensional cylindrical coordinates with radial symmetry, a Dirac delta function of strength $p$ is applied at the point and time of origin of the source, $I(r, 0)=p \delta(r)$.

The solution of Eq. (6) for a point source of strength $p$ at $r=0$ may then be written

$I(r, t)=\frac{p}{4 \pi D_{i} t} \exp \left(\frac{-r^{2}}{4 D_{i} t}\right)$.

This expression may be used for each of the inhibitor sources (sprouts), and summed so that the general expression for the inhibitor concentration within the domain may be written as

$I(r, t)=\sum_{n=1}^{N_{s}} \frac{p}{4 \pi D_{i}\left(t-t_{n}\right)} \exp \left(-\frac{\left(r-r_{n}\right)^{2}}{4 D_{i}\left(t-t_{n}\right)}\right) H\left(t-t_{n}\right)$,

where $H$ is the Heaviside function, and we consider that there are $N_{s}$ sprouts, and sprout $n$ is initiated at time $t_{n}$, position $r_{n}$. There is no steady state solution to the inhibitor equation.

In this study, we have assumed that the inhibitor production occurs as soon as the appropriate conditions have been met. It may well be that there is a delay before the inhibitor is produced, and this point is mentioned in Section 7. 


\subsection{Non-dimensionalisation}

We adopt the non-dimensionalisations:

$$
\begin{array}{ll}
r^{*}=\frac{r}{L}, & t^{*}=\frac{t}{\tau}, \quad \tau=\frac{L^{2}}{4 D_{a}}, \quad A^{*}=\frac{A}{\hat{A}}, \quad \hat{A}=\frac{q \tau}{\pi L^{2}}, \\
I^{*}=\frac{I}{\hat{I}}, & \hat{I}=\frac{p}{\pi L^{2}}, \quad \beta=\frac{D_{i}}{D_{a}}, \quad \gamma=\lambda \tau,
\end{array}
$$

where $L$ is the shortest distance between tumour and blood vessel.

The scaling for $A$ may be thought of as an average of the concentration of activator released in time $\tau$, over a circular area of radius, $L$. Similarly, the scaling factor for $I$ represents an average concentration of the amount of inhibitor released by one sprout, spread over a circular area of radius $L$.

$\tau$, the non-dimensionalisation for time, has been chosen for convenience, but corresponds with the definition of root mean squared length of diffusion in two dimensions, $4 D t$ (given in Segel, 1980): thus $L^{2}$ would be the root mean squared distance a particle diffuses over time $\tau$, in a twodimensional system.

Applying these transformations, the non-dimensionalised expressions for activator and inhibitor may be rewritten as

$A^{*}\left(r_{a}^{*}, t^{*}\right)=\int_{0}^{t^{*}} \frac{1}{u} \exp \left(\frac{-r_{a}^{* 2}}{u}-\gamma u\right) \mathrm{d} u$

and

$I^{*}\left(r^{*}, t^{*}\right)=\sum_{n=1}^{N_{s}} \frac{1}{\left(t^{*}-t_{n}^{*}\right)} \beta \exp \left(-\frac{\left(r^{*}-r_{n}^{*}\right)^{2}}{t^{*}-t_{n}^{*}} \beta\right) H\left(t^{*}-t_{n}^{*}\right)$,

where $\beta=D_{a} / D_{i}$ is the ratio of diffusion coefficients, and $\gamma=\lambda_{a} \tau$. Scaling the activator and inhibitor values by their respective non-dimensionalised source strengths reduces the number of parameter estimations required for the model. Continuing with the nondimensionalisation, expression (3) for activator concentration under conditions of no decay, may be rewritten as

$A^{*}\left(r_{a}^{*}, t^{*}\right)=E_{1}\left(\frac{r_{a}^{* 2}}{t^{*}}\right)$.

The non-dimensionalised expression for the activator steady state (with decay) may be written as

$A^{*}\left(r_{a}^{*}\right)=2 K_{0}\left(r_{a}^{*} \sqrt{4 \gamma}\right)$.

Note that $r^{*}$ is measured from the position of the first sprout, $r_{a}^{*}$ is measured from the tumour, and they are related in the non-dimensional system by $r_{a}^{* 2}=1+r^{* 2}$. For the remainder of the paper, star notation will be dropped, and distances will be expressed as a function of $r$, the nondimensionalised (linear) distance along the blood vessel from the position of the first sprout or midpoint.

\subsection{Parameter values}

Values or estimates are required for the parameters $\beta, \gamma$, for the (scaled) trigger levels of both activator and inhibitor, and for values of $L$ and $D_{a}$, used in the nondimensionalisation. For the purposes of this paper we chose $L=2 \mathrm{~mm}$, which is consistent with the experiment described in Section 2.

\subsubsection{Ratio of diffusion coefficients, $\beta$}

Finding an accurate estimate for the ratio of diffusion coefficients is somewhat difficult. An initial estimate can be made based on the molecular weights of the species involved. Use of the Einstein-Stokes formula (Shaw and Williams, 2003) for calculating diffusivity of a molecule in solution would lead to the ratio of diffusion coefficients of the two molecules being inversely proportional to the ratio of their hydrodynamic radii. We assume that the hydrodynamic radius is approximately proportional to the cuberoot of the molecular weight $(m)$. The ratio of the diffusion coefficients of inhibitor to activator is then proportional to $\sqrt[3]{m_{a} / m_{i}}$. Endostatin has a molecular weight of around $22 \mathrm{kDa}$, tumstatin $28 \mathrm{kDa}$, and angiostatin $57 \mathrm{kDa}$. Although VEGF has several different isoforms, the most commonly found active forms have molecular weights ranging from 34 to $45 \mathrm{kDa}$ (Dvorak, 2002). Using these molecular weights would suggest $\beta$ values ranging from 0.5 to 1.2 , approximately. Many other factors could impact the ratio of diffusion coefficients, and for these reasons a range of values for $\beta$ has been investigated. As well as finding the ratio of diffusion coefficients, we require an estimate for the diffusion coefficient of the activator, $D_{a}$, in order to estimate the characteristic time, $\tau$. We have used a value of $0.864 \mathrm{~mm}^{2} /$ day $\left(10^{-7} \mathrm{~cm}^{2} / \mathrm{s}\right)$ for $D_{a}$ based on the figure calculated by Ambrosi et al. (2005) using the EinsteinStokes formula. Although Mac Gabhann and Popel (2005) use a slightly higher value in their paper, our predictions are not sensitive to the choice of this parameter.

\subsubsection{Activator decay rate, $\gamma$}

An initial analysis of the model is made without decay of the activator $(\gamma=0)$, and this yields some interesting results. In this version of the model, however, the activator level at the midpoint shows monotonic increasing behaviour for all time. Thus any chosen activator level will eventually be met, and hence the model without decay cannot predict a physical limit to the distance between tumour and limbus for initiation of sprouting, although such a limit is a notable feature of corneal pocket angiogenesis experiments. This point is well illustrated in the paper by Folkman and Klagsbrun (1975) which examines the angiogenic and immune system responses to the implantation of a fragment of Brown-Pierce carcinoma in the cornea of a rabbit. Diagrams from the publication, which are reproduced in Fig. 3, show growth and regression of both tumour and neovasculature in an animal not previously immunized against this carcinoma. We use 

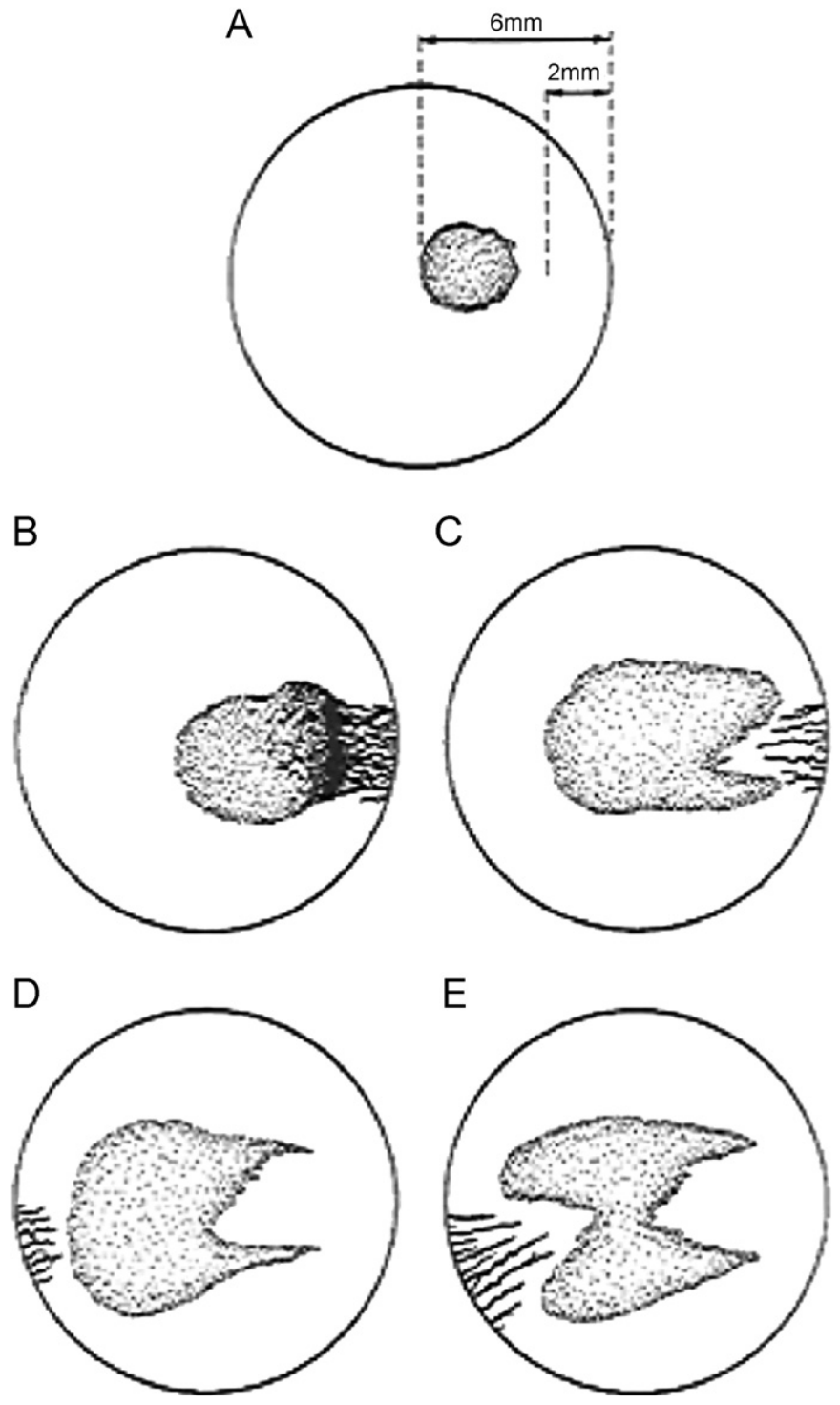

Fig. 3. Sequence of vascularisation of a tumour implanted into a rabbit cornea. Due to the immune system response to the tumor, two separate stages of vascularisation occur, shown in (B) and (D), both restricted to an area of the limbus closest to the tumour mass. Regression of the tumour shown in stages (C) and (E) is caused, in this case, by the immune system, and is not relevant to this study, but is included for completeness. Reprinted from Folkman (1974) with permission from Elsevier.

this experiment as a reference, not for the immune system response, but because during the process, formation of an angiogenic network occurred twice. In either case angiogenesis was restricted to an area of the limbus closest to the current tumour mass, and most importantly, Folkman and Klagsbrun report that angiogenesis did not occur at any stage of the experiment until an edge of the tumour had grown to within $2 \mathrm{~mm}$ of the limbus.

Restriction of angiogenesis to a portion of the limbus may be modelled by the inclusion of activator decay. In this model the concentration of the activator protein will reach a steady state value throughout the domain. If this value is lower than the activation level at all points along the limbus, sprouting will not occur at all, a point to which we will return later.

It is likely, therefore, that activator decay plays an important role in determining the patterns of angiogenic sprouting. An estimate for the value of the decay coefficient, $\lambda_{a}$, has been calculated from an in vitro halflife of VEGF, found by Serini et al. (2003) to be $64 \pm 7 \mathrm{~min}$. Behaviour of the model for a range of decay rates around this value has been investigated and is discussed in Section 5. Fig. 4 shows that activator levels are closely coupled to the decay rate: halving or doubling the decay rate changes activator levels along the limbus by more than an order of magnitude.

\subsubsection{Activator trigger level}

As discussed previously, angiogenic sprouting does not spread around the entire limbus, but is confined to an area of the limbus on either side of the site of the first sprout. The steady-state level of activator at the outermost sprout may therefore be taken to be the lowest level of activator for which spouting may occur, and this may be used as the activator trigger level. Fig. 4 may be used to find the appropriate activator trigger level for each of the given decay rates, having chosen a limit for sprouting. This method of choosing the activator trigger level does not work when the model is considered without decay (there is no activator steady state). In this case an estimate for the activator trigger level may be made using experimental data on time until appearance of the first sprout. By the time the sprout is visible, the activator level at the midpoint should have passed the trigger level (due to a lag between reaching the trigger level and a sprout becoming visible), and an estimate for the trigger level may be made. However, having estimated the time of emergence of the first sprout, calculations of subsequent patterns of sprouting may be made without finding the activator trigger level, using Eq. (13).

\subsubsection{Inhibitor threshold}

Values for the inhibitor threshold level may be chosen to give different regimes of sprouting along the limbus, and a number of these are discussed in the results section.

\section{Analysis of model with no decay}

\subsection{Simplification of sprouting conditions}

Neglecting decay of the activator enables an interesting preliminary analysis of the model. The analysis is greatly simplified by firstly showing that sprouts only form at points where concentrations of both activator and inhibitor are equal to their respective trigger levels, i.e. that the inequalities of the conditions for sprouting may be changed to equalities.

Clearly the first sprout forms as soon as $A=A_{\text {trig }}$ at $r=0$. By symmetry, the second and subsequent sprouts will occur in pairs, equally spaced about the midpoint of 


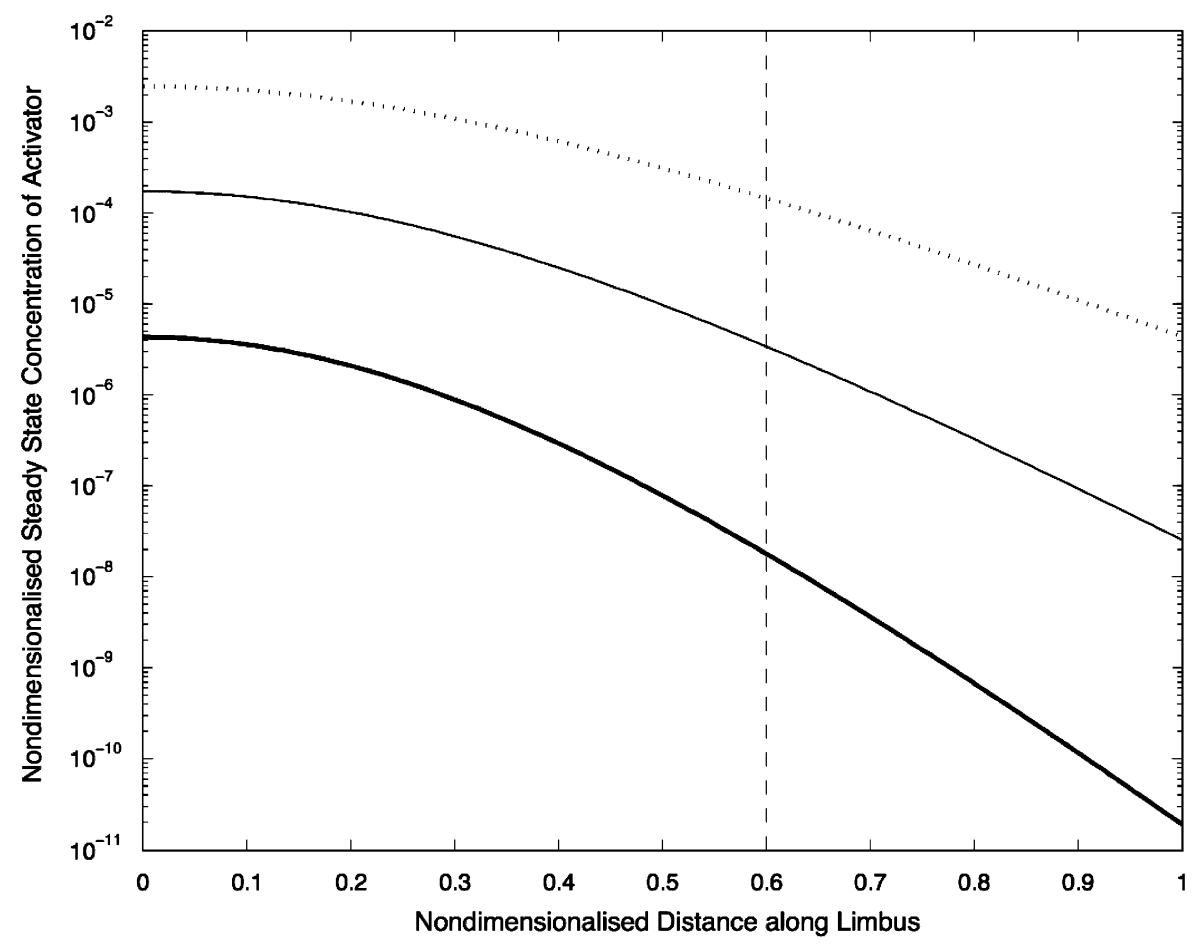

Fig. 4. Steady state non-dimensionalised activator levels (log scale) vs non-dimensionalised distance along limbus, $r$, for three different decay rates, $\gamma_{s}$ (light line), $\gamma_{s} / 2$ (dotted line), and $2 \gamma_{s}$ (heavy line), where $\gamma_{s}$ is the non-dimensionalised version of the decay rate given in Serini et al. (2003). The dashed vertical line may be used to find steady state values at a distance along the limbus equal to 0.6 of the distance from tumour to limbus. Note that changing the decay rate by a factor of two changes activator levels at any point on the limbus by more than an order of magnitude. As discussed in Section 3.3.3, this has a large impact on the chosen activator trigger level.

the domain. Therefore, for convenience, discussion will be focussed on events in the right (positive $r$ ) half of the domain unless otherwise noted. The second sprout will form at the first point on the limbus to satisfy both $A \geqslant A_{\text {trig }}$ and $I \leqslant I_{\text {thresh }}$, and we show graphically that this occurs when both $A=A_{\text {trig }}$ and $I=I_{\text {thresh }}$. In Fig. 5, we have $\hat{t}=t-t_{1}$ as the ordinate and $r$ as the abscicca. The area for which $(r, \hat{t})$ satisfies $A \geqslant A_{\text {trig }}$ is hatched, and the area for which $(r, \hat{t})$ satisfies $I \leqslant I_{\text {thresh }}$, is shaded. Each of these areas is bounded by a line showing the appropriate contour, $A=$ $A_{\text {trig }}$ and $I=I_{\text {thresh }}$. For sprouting we require the minimum ordinate $(\hat{t})$ value for which the hatched and shaded areas overlap, and this clearly occurs where the contour lines intersect, that is where $A=A_{\text {trig }}$ and $I=I_{\text {thresh }}$.

This finding may be extended to the position of the third and further sprouts, and to the model with activator decay included.

Once this property of the system is established, the activator constraint for sprouting under conditions without decay may be rewritten as follows:

$E_{1}\left(\frac{r_{a}^{2}}{t}\right)=A_{\text {trig }}$.

Since $E_{1}(x)$ is a single-valued function for positive reals, all solutions for which $A=A_{\text {trig }}$ correspond to the same value of $r_{a}^{2} / t$. This value may be found using the time of emergence of the first sprout $\left(t_{1}\right)$, since it occurs at a known location (the midpoint), where $r_{a}=1$. Thus, the activator condition may be further simplified to

$\frac{r_{a}^{2}}{t_{n}}=\frac{1}{t_{1}}$.

\subsection{Second and further sprouts, no decay}

The position and time of formation of the second sprout may be found analytically since at this stage there is only one source of inhibitor (the first sprout), so the inhibitor condition for sprouting reduces to

$I_{\text {thresh }}=\frac{\beta}{\hat{t}_{2}} \exp \left(-\frac{r_{2}^{2}}{\hat{t_{2}}} \beta\right)$,

where $\hat{t_{2}}=t_{2}-t_{1}$. Recalling that $r_{a}^{2}=1+r^{2}$, Eq. (13) may be manipulated to give $r_{2}^{2} / \hat{t}_{2}=1 / t_{1}$, and this may be substituted into Eq. (14). The position of the second sprout may then be found by solving

$r_{2}=\sqrt{\frac{\beta}{t_{1} I_{\text {thresh }}} \exp \left(\frac{-\beta}{t_{1}}\right)}$.

As a visualisation of the solution space after formation of the first sprout and before formation of the second, Fig. 6 shows contours of the concentration of inhibitor from the first sprout, in the $r-\hat{t}$ plane (with $\beta=1.0$ ). Overlaid on this plane are three curves of constant $1 / t_{1}$, each curve corresponding to $(r, \hat{t})$ pairs that satisfy the activator condition for a given value of $t_{1}$. The second sprout is 


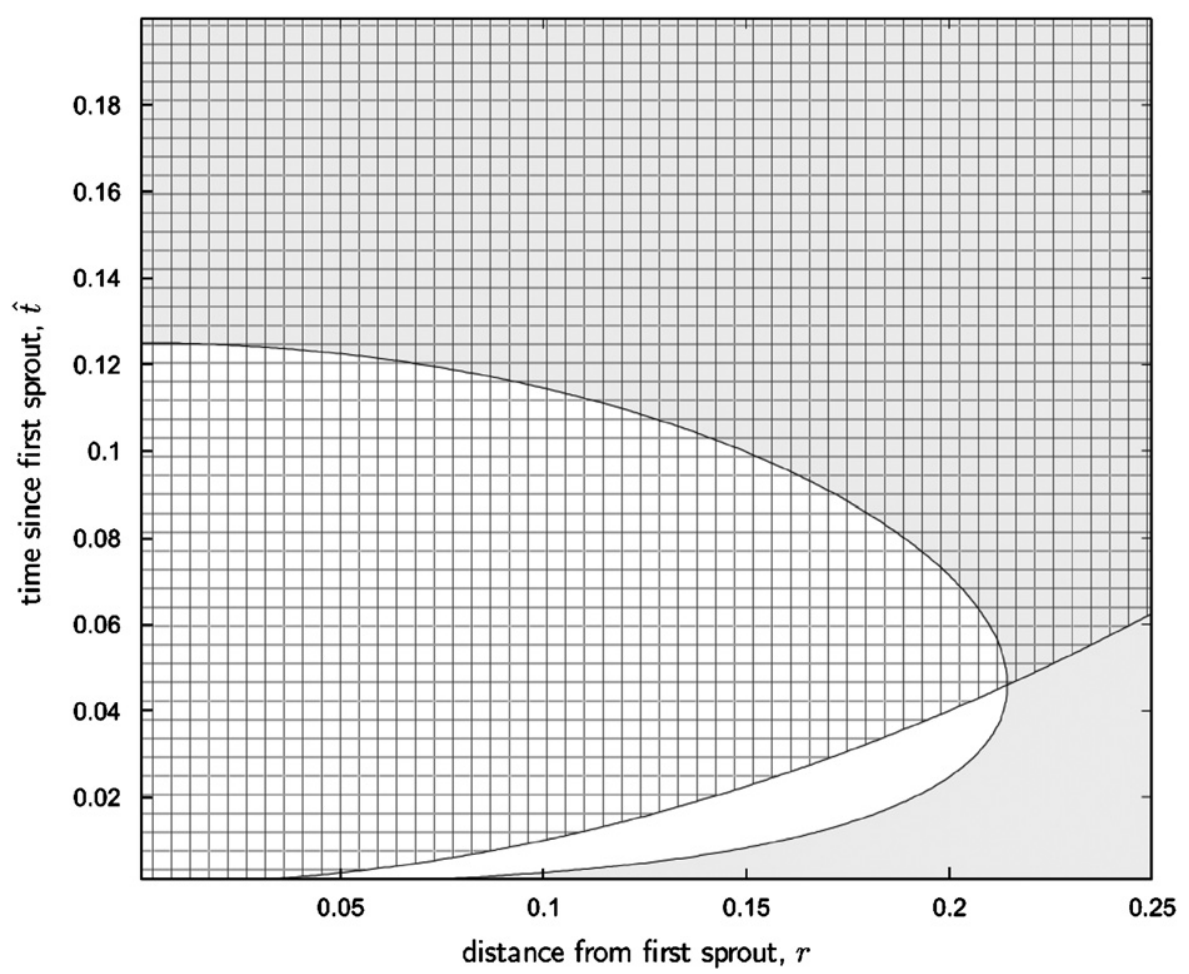

Fig. 5. The hatched area shows distribution in the $r-\hat{t}$ plane of activator levels greater than $A_{\text {trig }}$-where the activator condition for sprouting is met. The shaded area shows distribution of inhibitor levels less than $I_{\text {thresh }}$ - where the inhibitor condition for sprouting is met (inhibitor levels from the first sprout only). Any area of overlap fulfills both conditions, and this happens for the first time at a point where $A=A_{\text {trig }}$ and $I=I_{\text {thresh }}$. In this figure, $\beta=1$ and $t_{1}=1$. The ordinate value used is time since formation of the first sprout $t-t_{1}$, denoted $\hat{t}$. This figure was generated using Eqs. (12) and (9) with $n=1$.

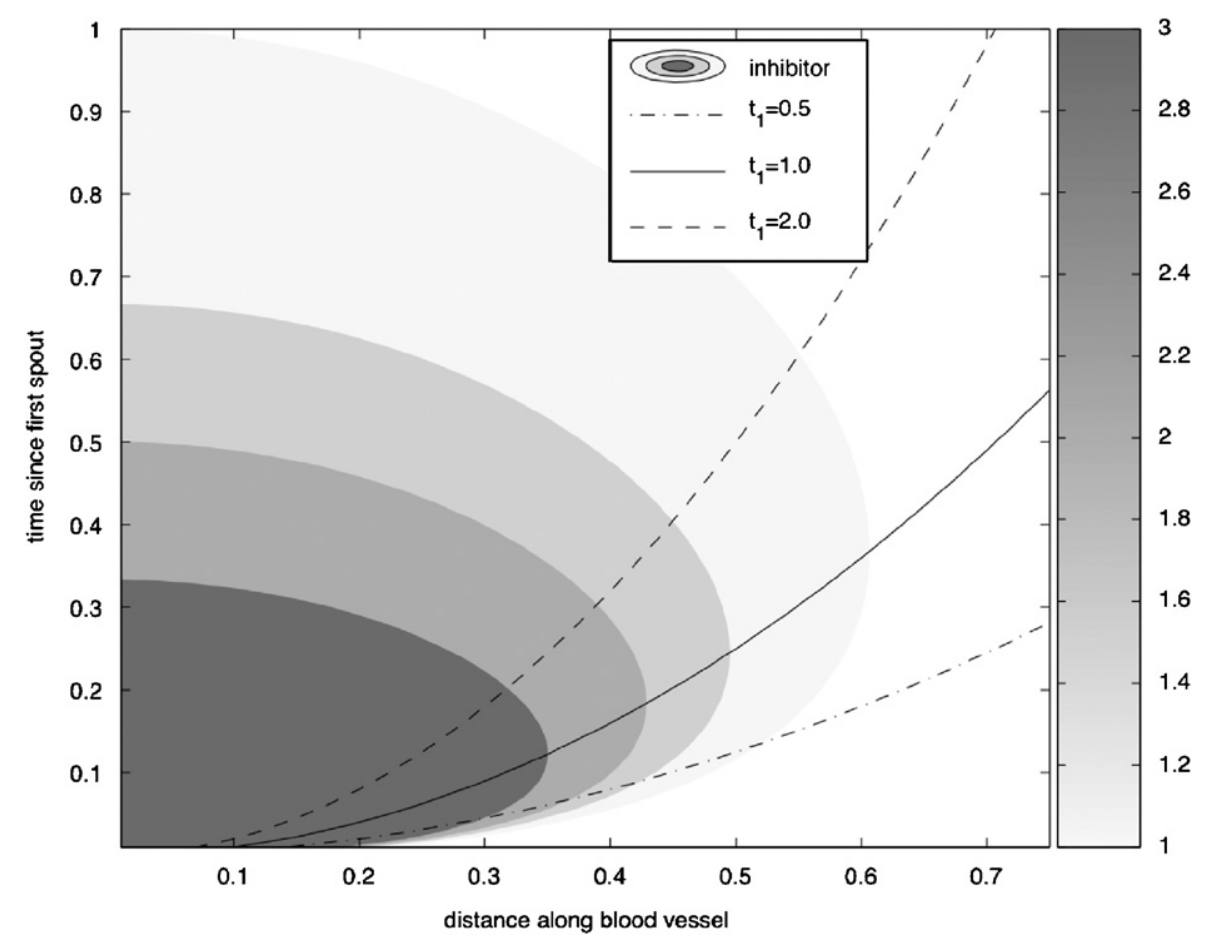

Fig. 6. Contours of inhibitor from the first sprout plotted in the $r$ vs $\hat{t}$ plane, for $\beta=1.0$. Overlaid on this plot are curves of constant $1 / t_{1}$, along which the activator condition for sprouting will be met (for the model without activator decay). The second sprout is predicted to form when the appropriate $1 / t_{1}$ line intersects the contour corresponding to the chosen inhibitor threshold value, $I_{\text {thresh }}$. This figure was generated using Eqs. (13) and (9) with $n=1$. 
predicted to form at a point in this plane where the appropriate $1 / t_{1}$ curve crosses the contour of the chosen inhibitor threshold value. Further sprouts will occur along curves of constant $1 / t_{1}$, but the inhibitor contours will change on addition of each new sprout. Solutions for further pairs of sprouts are found numerically.

\section{Analysis of the model with activator decay}

As discussed in Section 3.3.2, it is likely that decay of the activator plays a significant role in determining the patterns of angiogenic sprouting. The decay rate reported by Serini et al. (2003) is large enough to make a significant difference to our analysis. With $\gamma>0$, the expression for activator concentration, Eq. (8) has no closed form solution. Thus the $E_{n}$ function solution and the finding that sprouts will occur along curves of constant $r_{a}^{2} / t$ are both invalid for the model in this form. However, once the time for emergence of the first sprout, $t_{1}$, is found, the ratio $r_{a, n}^{2} / t_{n}$ for each further sprout will always be less than $1 / t_{1}$, where $r_{a, n}$ is the distance from the tumour to the point of emergence of sprout $n$. This relation may be used to calculate the minimum time to sprouting at any point on the limbus.

\subsection{Activator concentration}

Although a closed form solution for the integral in Eq. (8) has not been found, we can find the exact solution to the integral for one, quite useful, value of $t$. This solution comes from an interesting property of the integral which may be demonstrated as follows: using the substitution $w=r_{a}^{2} / \gamma u$ and dropping star notation, we may transform Eq. (8) to obtain

$$
\int_{r_{a}^{2} / \gamma t}^{\infty} \frac{1}{w} \exp \left(-\frac{r_{a}^{2}}{w}-\gamma w\right) \mathrm{d} w .
$$

Since choice of the integration variable, $w$, is arbitrary, we may replace it with $u$, and write

$\int_{0}^{t} \frac{1}{u} \exp \left(-\frac{r_{a}^{2}}{u}-\gamma u\right) \mathrm{d} u=\int_{r_{a}^{2} / \gamma t}^{\infty} \frac{1}{u} \exp \left(-\frac{r_{a}^{2}}{u}-\gamma u\right) \mathrm{d} u$.

Thus the amount of activator at any location, $r_{a}$, at a given time, $t$, is equal to the amount of activator which will accumulate at that location between time $r_{a}^{2} / \gamma t$ and $u=\infty$, the steady state. It follows that at any location, $r_{a}$, at time $t=r_{a} / \sqrt{\gamma}$,

$\int_{0}^{r_{a} / \sqrt{\gamma}} \frac{1}{u} \exp \left(-\frac{r_{a}^{2}}{u}-\gamma u\right) \mathrm{d} u=\int_{r_{a} / \sqrt{\gamma}}^{\infty} \frac{1}{u} \exp \left(-\frac{r_{a}^{2}}{u}-\gamma u\right) \mathrm{d} u$,

and so the level of activator will have reached half of its steady-state value. Our steady-state value is in the form of a modified Bessel function (see Eq. (11)), so we obtain the following exact relation for activator level at $t=r_{a} / \sqrt{\gamma}$,

$A\left(r_{a}, t=\frac{r_{a}}{\sqrt{\gamma}}\right)=K_{0}\left(r_{a} \sqrt{4 \gamma}\right)$.

It also follows from relation (16) that an approximation (or value for) the integral at any time, $t$, will also yield an approximation or value for the integral at time $r_{a}^{2} / \gamma t$, which may be expressed as follows:

$A\left(r_{a}, t\right)=\bar{A}\left(r_{a}\right)-A\left(r_{a}, \frac{r_{a}^{2}}{\gamma t}\right)$,

where $\bar{A}\left(r_{a}\right)$ is the steady-state value for the activator at $r_{a}$.

We have found the following relationships to be useful approximations to the activator concentrations for our parameter space, and leave discussion of the derivation of these ansätze to the Appendix.

For $t>r_{a} / \sqrt{\gamma}$, we use

$A\left(r_{a}, t\right)=\bar{A}\left(r_{a}\right)\left(\frac{1}{1+\mathrm{e}^{-2 z}}\right)$,

where $z=\sqrt{\gamma \sqrt{\gamma} / r_{a}}\left(t-r_{a} / \sqrt{\gamma}\right)$.

For $t<r_{a} / \sqrt{\gamma}$, we apply Relation (18) to Eq. (19), to give

$A\left(r_{a}, t\right)=\bar{A}\left(r_{a}\right)\left(1-\frac{1}{1+e^{-2 y}}\right)$,

where $y=\sqrt{r_{a} \sqrt{\gamma}}\left(r_{a} / \sqrt{\gamma} t-1\right)$.

\section{Results}

The model both with, and without, decay is able to predict a variety of sprout formation patterns. However, since it is clear that decay of the activator plays an important role in restricting the extent of sprouting, discussion of results will focus on the model with decay. Figs. 7 and 8 illustrate typical sprout positions part-way through both a solution to the model with activator decay, and a solution to the model without decay. The value of $t_{1}$, the time of the first sprout, is the same for both simulations. The shaded area shows positions that satisfy both activator and inhibitor conditions for sprouting, with the contribution to local inhibition from the most recent sprouts showing as circular indentations in the contour (more obvious in Fig. 7). We include animations of these figures over time as supplementary material.

\subsection{Variation of parameters, model with activator decay}

Different sprouting patterns may be obtained by variation of the model parameters. The condition for no sprouting at all to occur is achieved simply by setting the activator trigger level to a value less than the activator steady state value at the midpoint. With the activator trigger level set above this value, the model will always give a first sprout at the midpoint. Depending on other parameter values, the next sprout may occur anywhere from very close to the midpoint to a point very close to the 


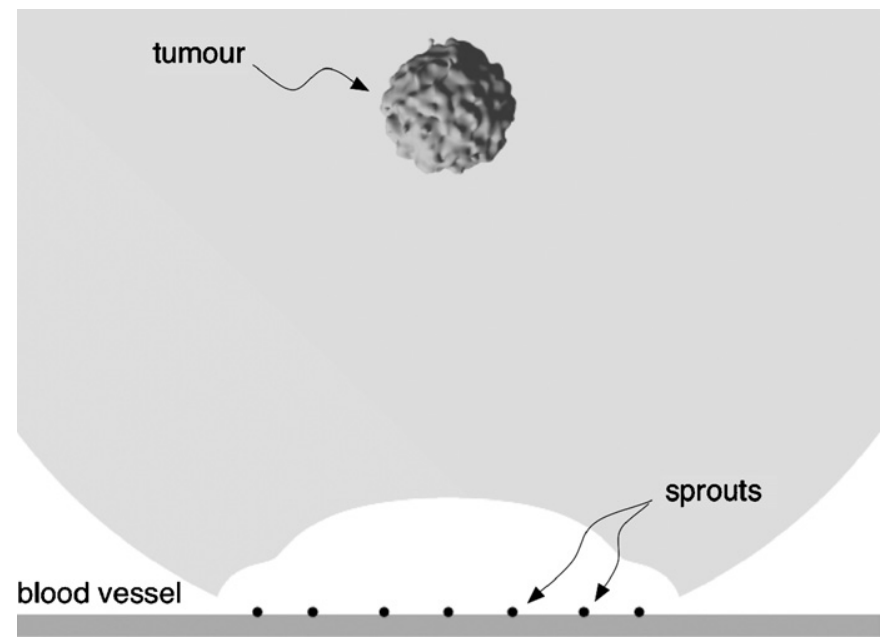

Fig. 7. Model with activator decay (Eqs. (8) and (9)). Sprout positions (black dots) and area satisfying both activator and inhibitor condition (shaded light grey) at non-dimensionalised $t=0.430$. A new sprout is predicted to form when the shaded area contacts the blood vessel (horizontal grey line). Non-dimensionalised parameter values are $\gamma=10$, $\beta=1.0, I_{\text {thresh }}=20$, sprouting radius of 0.5 . First sprout appears at $t_{1}=0.30306$.

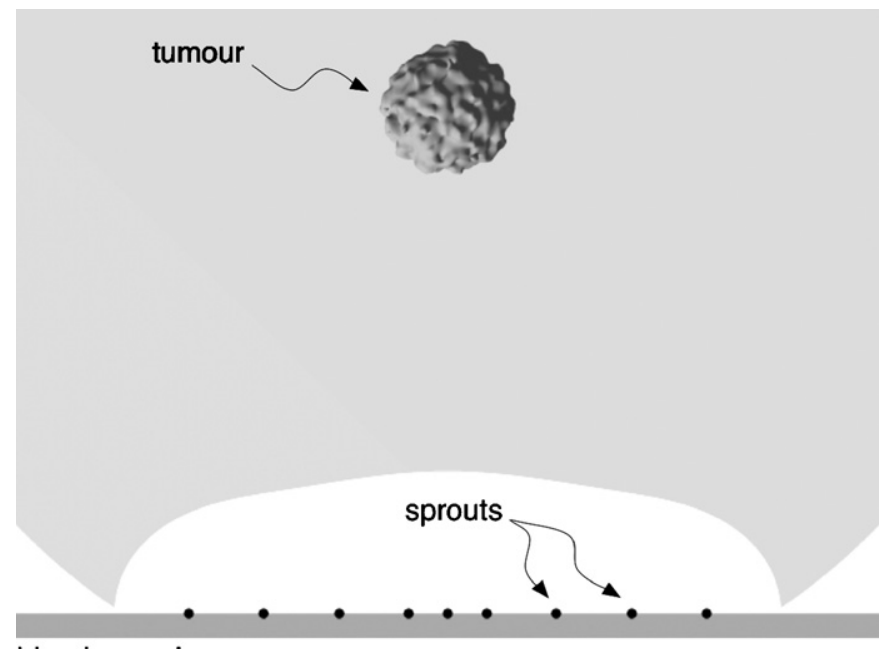

blood vessel

Fig. 8. Model without activator decay (Eqs. (9) and (10)). Sprout positions (black dots) and area satisfying both activator and inhibitor condition (shaded light grey) at non-dimensionalised $t=0.430$. A new sprout is predicted to form when the shaded area contacts the blood vessel (horizontal grey line). Non-dimensionalised parameter values are $\beta=1.0$, $I_{\text {thresh }}=20, t_{1}=0.30306$.

limit of sprouting. Fig. 9 shows sprouting positions for various sets of parameter values. In general, away from the limit of sprouting, the effect of increasing parameters is to decrease the inter-sprout spacing. The exception to this is the ratio of diffusion coefficients, $\beta$. As $\beta$ increases from very small values to values greater than 1 , the distance between sprouts increases, reaches a maximum, then decreases, even for sprouts close to the midpoint. However, we estimate that the ratio of diffusion coefficients will be of the order of unity, around which point the general trend is for decreasing inter-sprout distance with increasing $\beta$. Since inhibitor levels must be below the threshold for sprouting to occur, increasing the inhibitor threshold relaxes the condition on sprouting, and sprouts will form closer together. Increasing the decay rate whilst keeping the same limit of sprouting effectively decreases the required trigger level for sprouting, also making it easier for sprouts to occur, and hence sprouts form closer together as the decay rate increases. Increasing the limit of sprouting also directly decreases the activator trigger level, and, once again, sprouts will occur closer together as the limit of sprouting is increased.

In Fig. 9(d) clustering of sprouts at the limit of the domain, and occasionally near the midpoint, may be observed. Reasons for this and applicability of the model to small intersprout spacings is discussed in the following section.

\subsection{Long term behaviour of the model}

In this section we consider the long term distributions of activator and inhibitor. We note that the initiation of sprouts during more advanced angiogenesis falls well outside the scope of our model, since molecular and cellular events, including changes in the parent vessel and in the extracellular matrix, would introduce many new considerations. It may be that in fact, by the time the later sprouts are initiated, the capillaries generated from the early sprouts would have already reached the tumour, and the dynamics of angiogenic factor production would be affected. Our model would not be valid in that regime.

Our first note on the long term behaviour of the model concerns inhibitor levels in the middle of the domain. Inhibitor is produced in a discrete amount at each sprouting point, and is subject to diffusion over an infinite plane, so the contribution to inhibitor level from a single source will tend to zero. Sprouting begins at the midpoint and progresses outwards symmetrically. Inhibitor is produced from all sprouts, but with increasing time, the inhibitor concentration falls in the middle of the domain. In contrast to this, the activator concentration always increases, and consequently, there exists a $t$ such that $A \geqslant A_{\text {trig }}$ and $I \leqslant I_{\text {thresh }}$ again in the middle of the domain. The trend may be observed in the animations we include as additional material.

To avoid this problem in generating the current results, we have demanded that no new sprouts form between existing sprouts. Early on in the process, this condition is not required, since the inhibitor levels stay above the threshold for the parameter values chosen. We refrain from predicting when sprouts would form between existing sprouts since this is outside the framework of our current modelling.

Our second comment concerns imposing a lower limit to the intersprout spacing. In its current form the model has no restrictions on the spacing of sprouts, treating the limbal blood vessels as a single continuous entity. However, since blood vessels are composed of cells which will 
a

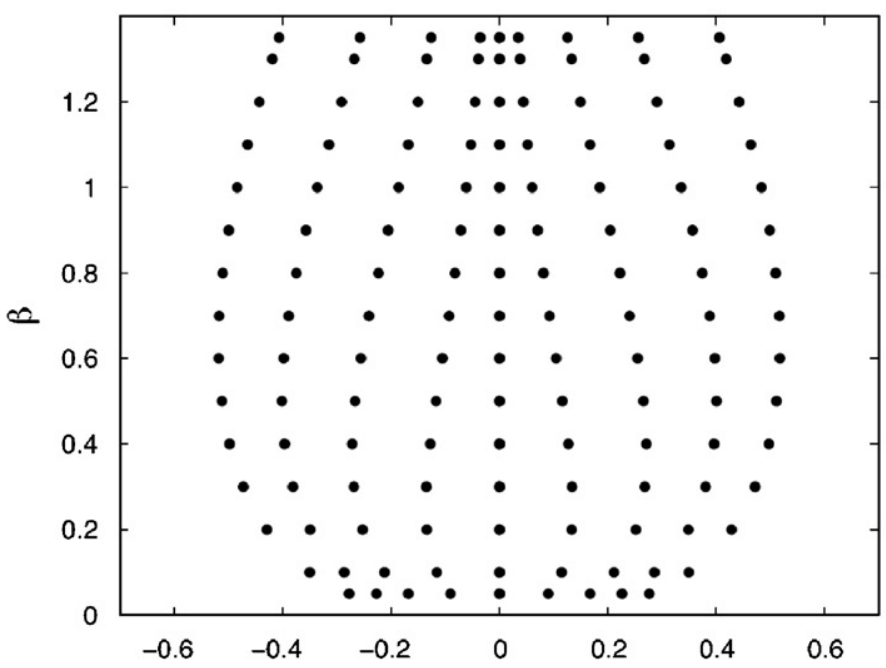

C

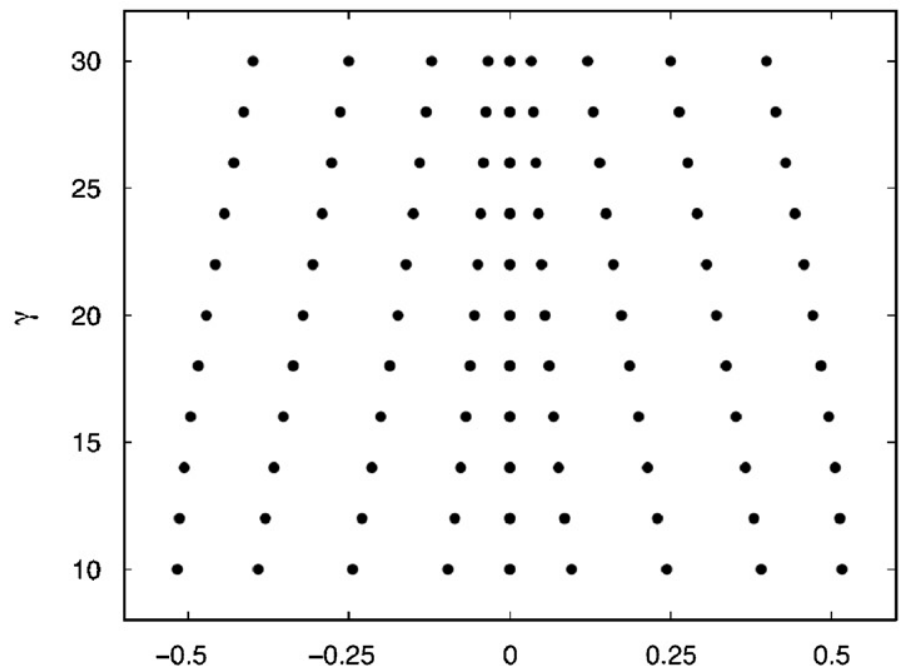

b

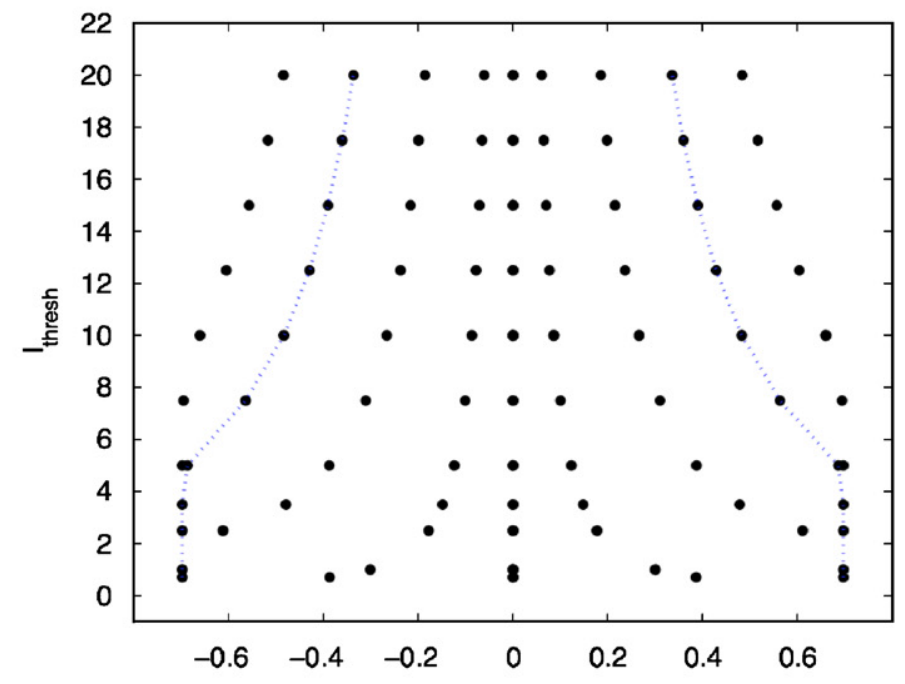

d

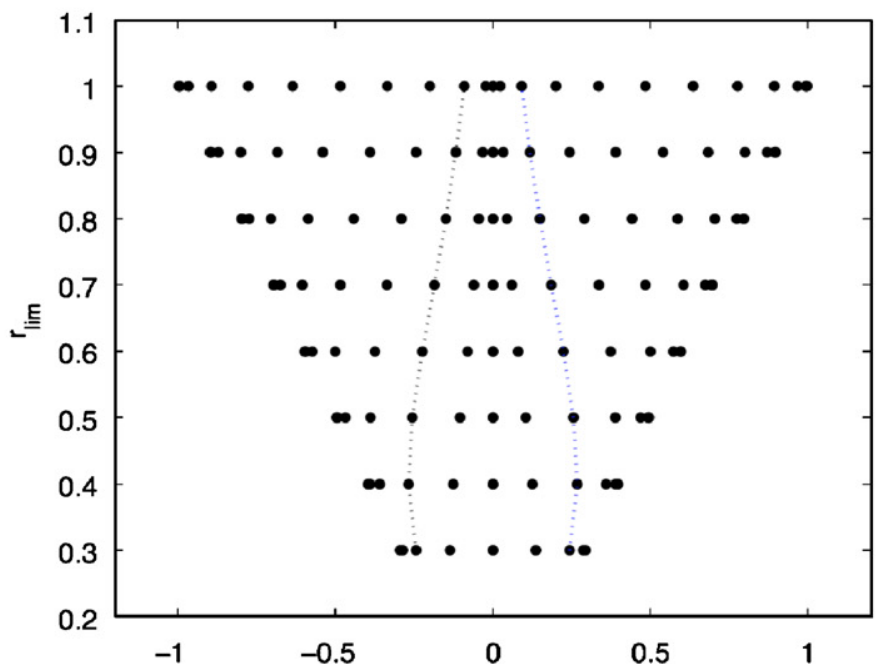

Fig. 9. Model with activator decay (Eqs. (8) and (9)). Variation of sprouting positions with model parameters. Black dots indicate predicted sprout positions. Dotted grey line in subfigure (b) follows the position of the third pair of sprouts as the inhibitor threshold varies. Dotted grey line in subfigure (d) follows the position of the second pair of sprouts as the limit of sprouting varies. Note that subfigure (d) shows sprouts up to the limit of sprouting $\left(r_{l i m}\right)$, while the other subfigures show the first 9 sprouts only. Base parameter values are as follows: $\beta=1.0, r_{l i m}=0.7, \gamma=\gamma_{s}$, and $I_{\text {thresh }}=20.0$. Except near the limit of sprouting, increases in $I_{\text {thresh }}$ or $\gamma$ and in the activator trigger level tend to result in decreases in the inter-sprout spacings, whilst the response to $\beta$ varies. (a) Ratio of diffusion coefficients; (b) inhibitor threshold; (c) activator decay rate; (d) activator trigger level.

react as discrete units to angiogenic stimuli, it follows that this model is not applicable to scales of the order of one endothelial cell length and below (less than approximately $10 \mu \mathrm{m}$ or $5 \times 10^{-3}$ in our non-dimensional system). This particularly applies when determining the position and time of the outermost pair of sprouts. Without applying this caveat, sprouting would continue indefinitely just short of the limit of sprouting. Once this condition is applied, sprouts may still cluster slightly at the limit of sprouting under some, but not all, parameter sets. (For example, there is clustering in Fig. 9(d), but not in Fig. 9(b) for low values of $I_{\text {thresh }}$ ).

We conclude this section with a discussion of the mathematics behind sprout spacings at the limit of sprouting in the absence of an imposed restriction to the intersprout spacing. For this it may be best to consider the progress of the activator trigger level contour along the blood vessel. Sprouts must occur at points where this contour intersects the blood vessel, and the slower the contour moves, the closer the sprouts will be spaced together (the inhibitor has time to diffuse away). In the Appendix we derive an expression to approximate the speed of the activator trigger level contour at the limbus as it approaches the limit of sprouting, finding that the speed of this contour is proportional to its distance from the limit of sprouting. Therefore, very close to the limit of sprouting, the speed of the contour along the blood vessel can become very slow indeed. Inhibitor produced by sprouts in this area always has sufficient time to diffuse away before the activator trigger level contour has moved far, giving rise to 
very closely spaced sprouts. According to this relationship (Eq. (A.7)), the activator trigger level contour would take infinite time to reach the limit of sprouting, and so sprouts will keep forming. This effect can also tend to decrease the inter-sprout spacing in the approach to the limit of sprouting, although this depends on other parameters and in particular on the number of sprouts already formed and hence the amount of inhibitor present.

\section{Conclusions and directions for future research}

We have shown that a simple mechanistic mathematical model with two, non-interacting, species can predict a variety of sprout initiation patterns. The model is able to show conditions under which no sprouting will occur (see Section 6.1), a notable feature of the experiments by Folkman and Klagsbrun (1975). Results may be used to provide initial capillary positions for more extensive models of angiogenesis, including those with a stochastic basis.

We conclude this section with a discussion of directions which may be taken to refine the model.

We have attempted to relate the spacings in the model to experimental data on spacings, but we have been unable to find directly relevant investigations. One testable prediction of this model is that if the tumour is close to the limbal vessel, then the sprouts will be spaced closer together. In addition, the model predicts that, if the limit of limbal sprouting was defined by a given activator concentration, then the closer the tumour is to the limbal vessel, the larger the sprouting region.

One of the most notable features of tumour-induced angiogenic sprouting is the formation of a dense region of new capillaries close to the tumour, known as the brush border. Anderson and Chaplain (1998) have developed a model for the generation of new sprouts in the brush border region, relating this to the age of the current sprout, the existence of sufficient space locally for a sprout to form, and a requirement that the endothelial sprout density is greater than a threshold level that is inversely proportional to the local attractant concentration. We note that such a brush border effect could be predicted with the present model naturally if the quantity of inhibitor produced by formation of new sprouts in this area were less than the quantity of inhibitor produced on formation of sprouts from the limbal vessels. For example, sprouts in this area would be forming on other, relatively newly formed blood vessels, rather than on the mature vessels of the limbus. Perhaps less basement membrane will have formed around these immature blood vessels, leading to a lower production of endostatin on sprouting. It is also possible that lower densities of plasma clotting products occur around the newer vessels (particularly since the flow of blood in these vessels can be very low), and consequently a reduction in plasminogen production could feasibly occur. Proposing specific mechanisms for a reduction in inhibitor products in the area of the brush border is highly speculative, particularly since we do not necessarily attribute inhibition of sprouting to any single product, however the possibility of such occurrences is not unreasonable.

We have greatly simplified the biology that is involved in the initiation of angiogenesis, and mention here a few of the processes that bear consideration for inclusion in the mathematical model. One such process is the removal of activator and inhibitor proteins by the existing vasculature. Both angiogenic and antiangiogenic products have been isolated from the circulation, and so it may be necessary to incorporate a sink for either or both species at the limbus. It is also worth considering whether uptake of these proteins by the endothelial cells themselves could be included in the mathematical model.

Another biological consideration is that of the involvement of receptor proteins in the cellular response to angiogenic events. It may be too simplistic to argue that activation of angiogenesis is linked to the local concentration of a protein. Perhaps the accumulation of a number of bound receptors acts as the trigger, and this may not correspond to the local concentration of the activator. The consideration of receptor binding opens a very large area for modelling including the examination of such processes as the upregulation of receptor expression by activated cells, internalization of bound receptors, and receptor kinetics.

It has been shown that VEGF accumulates in the tissue surrounding solid tumours, and such accumulation would affect the nature of our diffusion term. Leakage of plasma at the site of new capillary sprouts may also affect diffusion of proteins, and the clotting itself may act as a source for a non-diffusing type of local inhibition. All of these factors bear consideration for inclusion in the model.

Mathematically, modifications could include replacement of such physical impossibilities as the instantaneous point source, with, for example, a distributed, time dependent version. It would also be of interest to model a time-delay between activation of the endothelial cells and release of inhibitor proteins.

\section{Acknowledgements}

This work was carried out at Queensland University of Technology during a visit by PKM funded under the Visiting Fellowship Scheme. PKM was partially supported by a Royal Society Wolfson Merit Award. The authors are grateful to the anonymous referees for their helpful suggestions concerning this manuscript.

\section{Appendix A}

\section{A.1. Derivation of hyperbolic tangent ansätze}

To perform a mathematical analysis on the model with activator decay included, we considered a variety of standard techniques to approximate the integral in 
Eq. (8). The most elegant approximation, a hyperbolic tangent function, was suggested by the shape of the relation between activator concentration and time, plotted from quadrature results. A sample of these is shown in Fig. A.1. We use the following form for the hyperbolic tangent approximation,

$A\left(r_{a}, t\right)=0.5[1+\tanh \{m(t-n)\}] \bar{A}\left(r_{a}\right)$.

In this form, the activator concentration at $r_{a}$ reaches half of its steady-state value at time $t=n$, and at this point, $\partial A / \partial t=0.5 m \bar{A}\left(r_{a}\right)$. From the results in Section 5.1, we know that the activator concentration at any point, $r_{a}$, always reaches half of its steady state value at time $t=r_{a} / \sqrt{\gamma}$. We also know that, at $t=r_{a} / \sqrt{\gamma}$, $\mathrm{\partial} A / \partial t=\sqrt{\gamma} / r_{a} \exp \left(-2 r_{a} \sqrt{\gamma}\right)$. This gives us $n=r_{a} / \sqrt{\gamma}$, and $m=2 \sqrt{\gamma} \exp \left(-2 r_{a} \sqrt{\gamma}\right) / \bar{A}\left(r_{a}\right) r_{a}$. To simplify the value for $m$ we use the following approximation to the modified Bessel function:

$K_{0}(x) \approx \frac{\exp (-x)}{\sqrt{(2 / \pi) x}}$

for large $x$. This gives an approximation to the steady state value of

$\bar{A}\left(r_{a}\right)=\frac{\exp \left(-2 r_{a} \sqrt{\gamma}\right)}{\sqrt{r_{a} \sqrt{\gamma} / \pi}}$.

Substituting back into the expression for $m$ we get $m=2 \sqrt{\gamma \sqrt{\gamma} / r_{a} \pi}$, however we have found that this fits less well than choosing $m=\sqrt{\gamma \sqrt{\gamma} / r_{a}}$ due to differing curvatures of the two functions. The slightly lower value for $m$ has not been rigorously determined, but has been found to operate very well for our parameter space. Substituting these values for $m$ and $n$ into Eq. (A.1) gives us

$A\left(r_{a}, t\right)=0.5\left(1+\tanh \left(\sqrt{\frac{\gamma \sqrt{\gamma}}{r_{a}}}\left(t-\frac{r_{a}}{\sqrt{\gamma}}\right)\right)\right) \bar{A}\left(r_{a}\right)$,

and this approximation is shown in Fig. A.1 together with the results of quadrature.

Rewriting this using the exponential form for the hyperbolic tangent leads to Eq. (19), given in Section 5.1. This approximation works best for values above $t=r_{a} / \sqrt{\gamma}$, so we use Relation (18) to give an approximation for $t<r_{a} / \sqrt{\gamma}$, given by Eq. (20).

\section{A.2. Progress of activator trigger level contour along blood vessel}

To develop an expression for the speed of progress of the activator trigger level along the blood vessel we use the approximation, Eq. (19), and the approximation to the steady state given by Eq. (A.2). We use Eq. (19), which applies to the upper portion of the integral, since we are particularly interested in behaviour as the contour approaches the limit of sprouting, noting that this limits the following discussion to $t>r_{a} / \sqrt{\gamma}$.

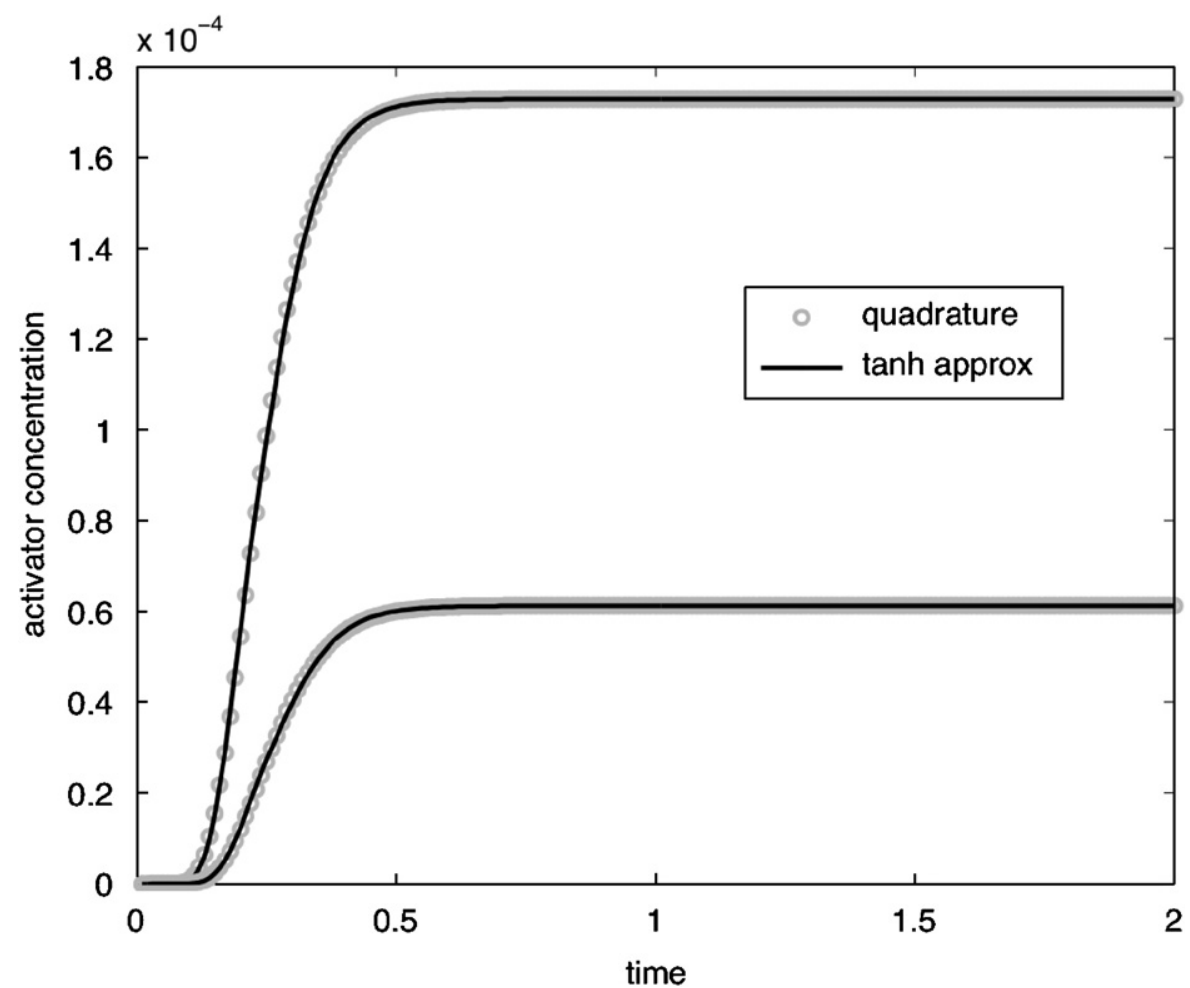

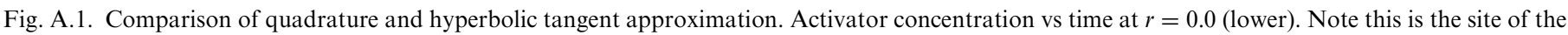

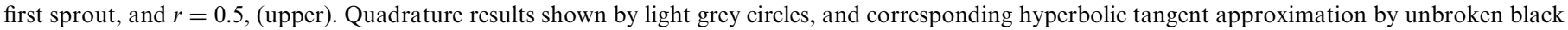
line. These results are for $\gamma=\gamma_{s}$. 
Substituting the activator trigger level into Eq. (19), and rearranging to find a relationship between time, $t_{c}$, and displacement, $r_{a c}$ for points that fall on the activator trigger level contour, we have

$t_{c}=\frac{r_{a c}}{\sqrt{\gamma}}-\frac{1}{2} \sqrt{\frac{r_{a c}}{\gamma \sqrt{\gamma}}} \ln \left(\frac{\bar{A}\left(r_{a c}\right)}{A_{\text {trig }}}-1\right)$.

Since the activator trigger level, $A_{\text {trig }}$, may be taken to be the activator steady-state value at the limit of sprouting (represented here by $\left.r_{\text {alim }}\right), A_{\text {trig }}$ may be replaced by $\bar{A}\left(r_{\text {alim }}\right)$. Using the exponential approximation to the modified Bessel function given by Eq. (A.2), we manipulate and rewrite

$\ln \left(\frac{\bar{A}\left(r_{a c}\right)}{A_{\text {trig }}}-1\right)=\ln \left(\exp \left(-2 \sqrt{\gamma}\left(r_{\text {alim }}-r_{a c}\right)\right) \sqrt{\frac{r_{a l i m}}{r_{a c}}}-1\right)$.

As the activator contour approaches the limit of sprouting, $r_{a c}$ approaches $r_{\text {alim }}$, so the fraction $\sqrt{r_{a l i m} / r_{a c}}$ becomes very close to unity, and may be disregarded. Also, since $\left(r_{\text {alim }}-r_{a c}\right)$ is small, we may approximate the exponential using a first-order Maclaurin series, giving

$\ln \left(\exp \left(-2 \sqrt{\gamma}\left(r_{\text {alim }}-r_{a c}\right)\right)-1\right) \approx \ln \left(-2 \sqrt{\gamma}\left(r_{\text {alim }}-r_{a c}\right)\right)$.

Our simplified expression for $t_{c}$ becomes

$t_{c}=\frac{r_{a c}}{\sqrt{\gamma}}-\frac{1}{2} \sqrt{\frac{r_{a c}}{\gamma \sqrt{\gamma}}} \ln \left(-2 \sqrt{\gamma}\left(r_{a l i m}-r_{a c}\right)\right)$.

This may be differentiated to find

$$
\begin{aligned}
\frac{\partial t_{c}}{\partial r_{a c}}= & \frac{1}{\sqrt{\gamma}}-1 / 4 \ln \left(2 \sqrt{\gamma}\left(r_{a l i m}-r_{a c}\right)\right) \frac{1}{\sqrt{r_{a c} \gamma^{3 / 2}}} \\
& +1 / 2 \sqrt{\frac{r_{a c}}{\gamma^{3 / 2}}}\left(r_{a l i m}-r_{a c}\right)^{-1} .
\end{aligned}
$$

Close to the limit of sprouting, this expression is dominated by the last term. Hence, by dropping the first two terms and inverting, we have an expression indicative of the behaviour of the activator trigger level contour along the blood vessel as $r_{a c}$ approaches $r_{\text {alim }}$

$\frac{\partial r_{a c}}{\partial t_{c}} \propto 2 \sqrt{\gamma \sqrt{\gamma}} \frac{\left(r_{a l i m}-r_{a c}\right)}{\sqrt{r_{a c}}}$.

In short, the closer the contour comes to $r_{\text {alim }}$, the slower it moves.

\section{Appendix B. Supplementary material}

Supplementary data associated with this article can be found in the online version at doi:10.1016/j.jtbi. 2007.08.030.

\section{References}

Ambrosi, D., Bussolino, F., Preziosi, L., 2005. A review of vasculogenesis models. J. Theoret. Med. 6, 1-19.

Anderson, A., Chaplain, M., 1998. Continuous and discrete mathematical models of tumour-induced angiogenesis. Bull. Math. Biol. 60, 857-900.
Araujo, R.P., McElwain, D.L.S., 2004. A history of the study of solid tumour growth: the contribution of mathematical modelling. Bull. Math. Biol. 66, 1039-1091.

Asahara, T., Chen, D., Takahashi, T., Fujikawa, K., Kearney, M., Magner, M., Yancopoulos, G., Isner, J., 1998. Tie2 receptor ligands, angiopoietin-1 and angiopoietin-2, modulate VEGF-induced postnatal neovascularization. Circ. Res. 83 (3), 233-240.

Clamp, A., Jayson, G., 2005. The clinical potential of antiangiogenic fragments of extracellular matrix proteins. Brit. J. Cancer 93, 967-972.

Dvorak, H.F., 2002. Vascular permeability factor/vascular endothelial growth factor: a critical cytokine in tumour angiogenesis and a potential target for diagnosis and therapy. J. Clin. Oncol. 20, 4368-4380.

Dvorak, H.F., 2003. How tumors make bad blood vessels and stroma. Am. J. Pathol. 162 (6), 1747-1757.

Felbor, U., Dreier, L., Bryant, R.A., Ploegh, H.L., Olsen, B.R., Mothes, W., 2000. Secreted cathepsin L generates endostatin from collagen XVIII. EMBO J. 19 (6), 1187-1194.

Folkman, J., 1974. Advances in Cancer Research, vol. 19. Elsevier Science and Technology Books.

Folkman, J., 2005. Antiangiogenesis in cancer therapy-endostatin and its mechanisms of action. Exp. Cell Res. 312 (5), 594-607.

Folkman, J., Klagsbrun, M., 1975. Fundamental Aspects of Neoplasia. Springer, Berlin.

Gazit, Y., 1996. Fractal Vasculature and Vascular Network Growth Modeling in Normal and Tumor Tissue. Ph.D. thesis, Massachusetts Institute of Technology, Whitaker College of Health Sciences and Technology.

Gimbrone, M., Cotran, R., Leapman, S., Folkman, J., 1974. Tumorgrowth and neovascularization - experimental model using rabbit cornea. J. Natl Cancer Inst. 52, 413-427.

Heljasvaara, R., Nyberg, P., Luostarinen, J., Parikka, M., Heikkila, P., Rehn, M., Sorsa, T., Salo, T., Pihlajaniemi, T., 2005. Generation of biologically active endostatin fragments from human collagen xviii by distinct matrix metalloproteases. Exp. Cell Res. 307, 292-304.

Jones, J., Glynn, P., Walker, R., 1999. Expression of mmp-2 and mmp-9, their inhibitors, and the activator $\mathrm{mtl}-\mathrm{mmp}$ in primary breast carcinomas. J. Pathol. 189, 161-168.

Jurasz, P., Alonso, D., Castro-Blanco, S., Murad, F., Radomski, M.W., 2003. Generation and role of angiostatin in human platelets. Blood 102 (9), 3217-3223.

Karihaloo, A., Karumanchi, S.A., Barasch, J., Jha, V., Nickel, C.H., Yang, J., Grisaru, S., Bush, K.T., Nigam, S., Rosenblum, N.D., Sukhatme, V.P., Cantley, L.G., 2001. Endostatin regulates branching morphogenesis of renal epithelial cells and ureteric bud. Proc. Natl Acad. Sci. 98 (22), 12509-12514.

Levine, H., Sleeman, B., Nilsen-Hamilton, M., 2001. Mathematical modeling of the onset of capillary formation initiating angiogenesis. J. Math. Biol. 42 (3), 195-238.

Mac Gabhann, F., Popel, A., 2005. Differential binding of VEGF isoforms to VEGF receptor 2 in the presence of neuropilin-1: a computational model. Am. J. Physiol.-Heart and Circulatory Physiol. 288 (6), 2851-2860.

Mantzaris, N.V., Webb, S., Othmer, H.G., 2004. Mathematical modeling of tumor-induced angiogenesis. J. Math. Biol. 49, 111-187.

Orme, M., Chaplain, M., 1996. A mathematical model of the first steps of tumour-related angiogenesis: capillary sprout formation and secondary branching. Math. Med. Biol. 13 (2), 73.

Ruegg, C., Hasmim, M., Lejeune, F.J., Alghisi, G.C., 2006. Antiangiogenic peptides and proteins: from experimental tools to clinical drugs. Biochim. Biophys. Acta (BBA)-Rev. Cancer 1765 , 155-177.

Saarela, J., Rehn, M., Oikarinen, A., Autio-Harmainen, H., Pihlajaniemi, T., 1998. The short and long forms of type xviii collagen show clear tissue specificities in their expression and location in basement membrane zones in humans. Am. J. Pathol. 153, 611-626. 
Segel, L., 1980. Mathematical Models in Molecular and Cellular Biology. Cambridge University Press, Cambridge.

Serini, G., Ambrosi, D., Giraudo, E., Gamba, A., Preziosi, L., Bussolino, F., 2003. Modeling the early stages of vascular network assembly. EMBO J. 22 (8), 1771-1779.

Shaw, D., Williams, R., 2003. Introduction to Colloid and Surface Chemistry. Elsevier Science.
Sim, B., 1998. Angiostatin and endostatin: endothelial cell-specific endogenous inhibitors of angiogenesis and tumor growth. Angiogenesis 2, 37-48.

Taraboletti, G., D’Ascenzo, S., Borsotti, P., Giavazzi, R., Pavan, A., Dolo, V., 2002. Shedding of the matrix metalloproteinases MMP-2, MMP-9, and MT1-MMP as membrane vesicle-associated components by endothelial cells. Am. J. Pathol. 160 (2), 673-680. 